\title{
Theme Issue on Hot and Dense Matter
}

\author{
Editors \\ BEDANGADAS MOHANTY \\ SOURENDU GUPTA
}

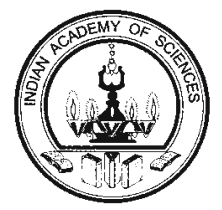

Published by

Indian Academy of Sciences

Bangalore 